\title{
An inverse approach to identifying the in vivo material parameters of female pelvic floor muscles using MRI data
}

\author{
YAO RuiWen ${ }^{1 \dagger}$, DU Le $^{2 \dagger}$, ZHU Lan $^{3 *}$, XIANG ZhiHai ${ }^{1 *}$, CHEN Cong ${ }^{4}, \mathrm{HOU} \mathrm{Bo}^{4}$ \\ \& FENG Feng ${ }^{4}$ \\ ${ }^{1}$ Key Laboratory of Applied Mechanics, Department of Engineering Mechanics, Tsinghua University, Beijing 100084, China; \\ ${ }^{2}$ Plastic Surgery Hospital (Institute), Chinese Academy of Medical Sciences \& Peking Union Medical College, Beijing 100144, China; \\ ${ }^{3}$ Department of Obstetrics, Peking Union Medical College Hospital, Beijing 100730, China; \\ ${ }^{4}$ Department of Radiology, Peking Union Medical College Hospital, Beijing 100730, China
}

Received June 1, 2014; accepted September 11, 2014; published online January 14, 2015

Citation: Yao RW, Du L, Zhu L, Xiang ZH, Chen C, Hou B, Feng F. An inverse approach to identifying the in vivo material parameters of female pelvic floor muscles using MRI data. Sci China Life Sci, 2015, 58: 305-308, doi: 10.1007/s11427-014-4771-6

Dear Editor,

Pelvic floor (PF) dysfunctions comprise of a wide spectrum of interrelated clinical conditions and represent an important health problem. The PF supporting system can be regarded as a loaded structure with a complex geometry that supports the increase of abdominal pressure and maintain normal position of pelvic organs (Figure 1A). The weakening of the PF supporting system is considered to be the cause of these PF dysfunctions. Recently, scientists have tried to use the finite element (FE) method to calculate the stress distributions to improve the biomechanical understandings in such structure [1].

The reliability of the material parameters of the PF muscles is crucial to the FE analysis. However, these material parameters are conventionally characterized through in vitro mechanical tests, which differ from those in the physiological or in vivo state. Therefore, people pay more attention to the in vivo biomechanical properties [2].

This paper develops a non-invasive inverse approach to identify the in vivo material parameters of the PF muscles. This approach tries to provide more realistic material parameters, thus may give more help to the numerical simula-

$\uparrow$ Contributed equally to this work

*Corresponding authors (email: zhu_julie@vip.sina.com; xiangzhihai@tsinghua.edu.cn) tions in relevant medical fields.

The isotropic incompressible neo-Hookean material model is used to describe the stiffness of the PF muscles with the constitutive equation [3]:

$$
\Phi=\frac{\mu}{2}(I-3)
$$

where $\Phi$ is the strain energy density; $\mu$ is the shear modulus; $I$ denotes the extent of the shape distortion. The parameters $\mu$ of the muscles need to be identified. For this purpose, a measuring process was designed to measure the intra-abdominal pressure (IAP), which is the agent of the PF muscle deformation. At the same time, the deformation data were acquired by a GE SIGNA EXCITE 1.5T HD MRI machine. All MRI data are the sagittal T2 weighted MRI images with a base resolution of $320 \times 288$ and $3.5 \mathrm{~mm}$ slice separation. Clinically, one well-accepted non-invasive technique to measure IAP is the intra-bladder pressure (IBP) monitoring through the patient's indwelling urinary Foley catheter, utilizing the bladder wall as a passive transducing membrane.

During the measuring, a 25-year-old nulliparous female volunteer lay in the MRI machine and tried to maintain her IAP at low and high levels by the visual feedback on the water column height inside the catheter connected to her bladder (Figure 1B). To ensure the effectiveness of IBP 

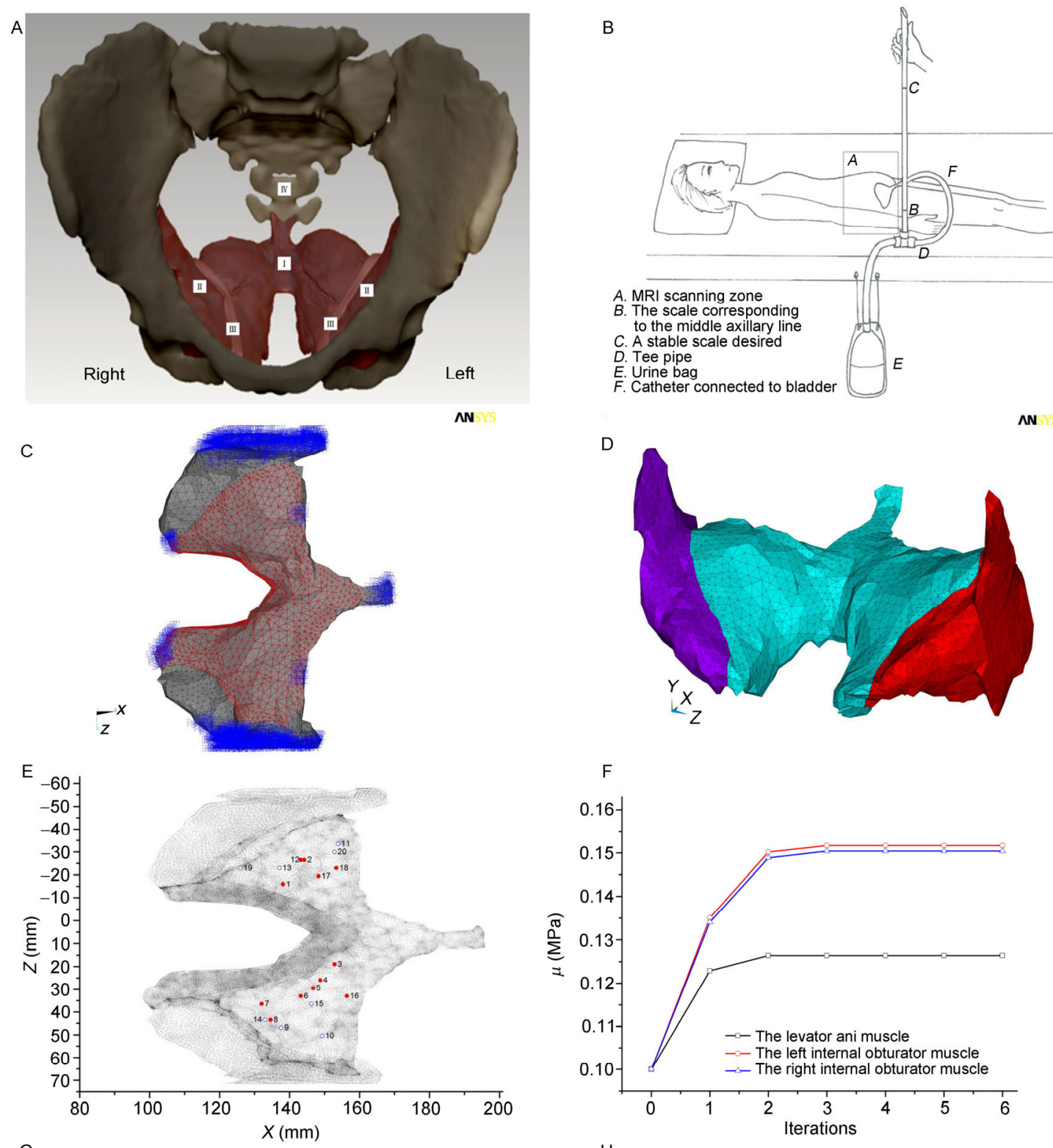

AN
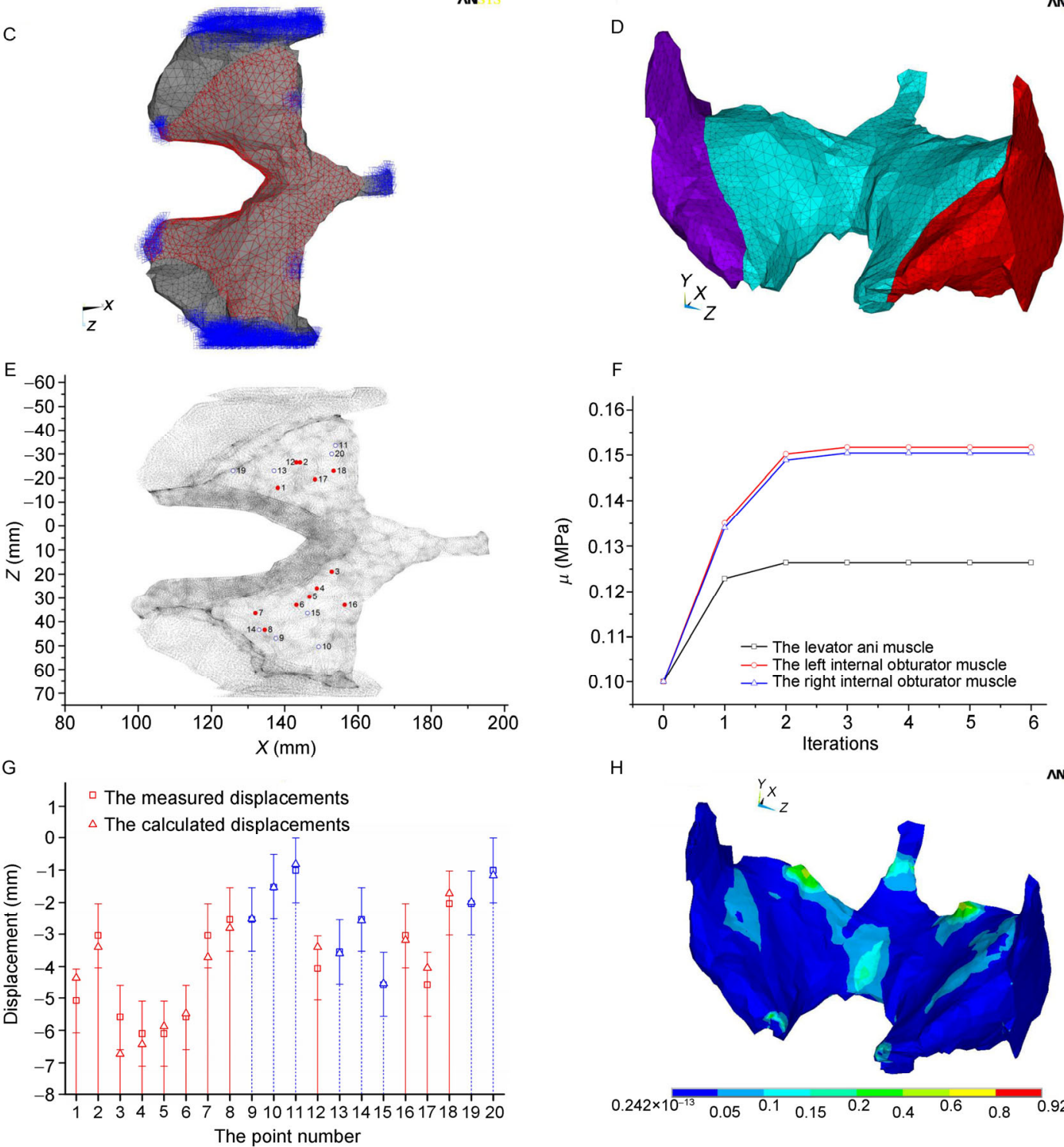

$\mathrm{H}$

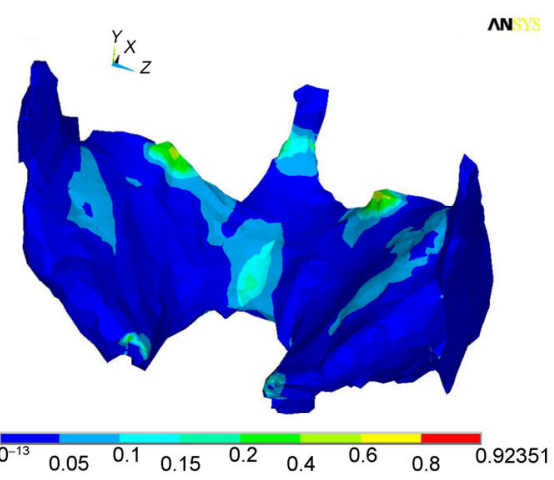

Figure 1 Illustrations of the muscle parameter identification approach. A, The PF structure: I, levator ani; II, internal obturator muscle; III, arcus tendineous; IV, coccyx. B, A sketch of the measuring equipment. C, The FE constraints and loads of the PF muscles. D, The FE model of the PF muscles (levator ani in the middle and the internal obturators on the sides). E, Top view of the distribution of the 20 measurement points. F, The identification procedure of the shear modulus. G, The comparison between the measured and calculated displacements of all measurement points. H, The distribution of von Mises stress $(\mathrm{MPa})$. 
measurement, about $50 \mathrm{~mL}$ physiological saline was injected into her bladder using a tee pipe. She would keep her abdomen relaxed at the lower IAP of $8 \mathrm{cmH}_{2} \mathrm{O}$ during the first set of MRI data acquisition, and then increased the contraction of abdomen at the higher IAP of $26 \mathrm{cmH}_{2} \mathrm{O}$ during the second set. The fluctuation of IAP was confined in a relatively narrow range (within $1 \mathrm{cmH}_{2} \mathrm{O}$ ). Thus the increase of IAP was $26-8=18 \mathrm{cmH}_{2} \mathrm{O}(1.764 \mathrm{kPa})$. This pressure increase is considered as the external load uniformly applied on the upper surface of the levator ani muscle (denoted by the red mesh at the center of Figure 1C).

The 3D morphological model of the PF muscles was reconstructed from the MRI images taken at the IAP of 8 $\mathrm{cmH}_{2} \mathrm{O}$ corresponding to the relaxed state. This model was then imported into ANSYS software to generate the FE mesh with the 10-node tetrahedral elements (Figure 1D). For simplification, tiny tissues like the tendons were considered a portion of the internal obturator muscles. This mesh contains about 77,000 nodes in total. And the front-ends of the internal obturator and the levator ani, the leftmost side of the left internal obturator, the rightmost side of the right internal obturator, the surface junction of the levator ani with the tailbone and two ischial spines were all fixed at the boundary (denoted by the blue triangular markers in Figure 1C).

One pair of feature points is defined as the same local micro-region of PF muscles before and after the deformation. The coordinate difference between the two points contains the local displacement information. This pair of points is also denoted as the 'displacement points' or 'measurement points'. The two sets of MRI images are compared slice by slice to discover the distinct feature points to produce sufficient displacement data. This searching depends on the clinical experiences in detecting the topological information in images. The displacement data was then obtained by the coordinate subtraction. Furthermore, these feature points could be matched with their corresponding mesh nodes in the FE model. However, the matching process would bring in extra errors because the mesh nodes are scattered in space rather than continuously distributed. To reduce the matching errors, the nearest FE node to each feature point was automatically picked out by computer with a tolerance of $1 \mathrm{~mm}$. Because of this tolerance, there only exist 20 qualified displacement points (Figure 1E). Since the obturator muscles in this model include tiny tissues, to ensure the data reliability all displacement points were sampled from the levator ani muscle, which is also the most important part. To increase data sensitivity, only the displacements along the height direction of the volunteer was used at each measurement point.

The identification method was programmed in a FORTRAN program to identify the muscle parameters. Since there were not so much a priori information, the conventional least-squares estimation method was employed, which can be solved iteratively through the Gauss-Newton method [4]:

$$
\boldsymbol{b}^{k+1}=\boldsymbol{G}\left(\boldsymbol{b}^{k}\right), \quad k=0,1,2, \ldots
$$

where $k$ denotes the iteration step; $\boldsymbol{b}$ is the parameter vector that contains the shear modulus of the levator ani, the left internal obturator and the right internal obturator; the operator $\boldsymbol{G}$ is a function of residual vector $\boldsymbol{R}$ and the Jacobian matrix $\boldsymbol{J}$ :

$$
\begin{gathered}
\boldsymbol{G}(\boldsymbol{b})=\boldsymbol{b}-\left[(\boldsymbol{J})^{\mathrm{T}} \boldsymbol{J}\right]^{-1}(\boldsymbol{J})^{\mathrm{T}} \boldsymbol{R}, \\
\boldsymbol{R}=\overline{\boldsymbol{u}}-\boldsymbol{S} \boldsymbol{u} \\
\boldsymbol{J}=\frac{\partial \boldsymbol{R}}{\partial \boldsymbol{b}}
\end{gathered}
$$

where $\overline{\boldsymbol{u}}$ and $\boldsymbol{u}$ are the measured and calculated displacements, respectively; $\boldsymbol{S}$ is the selective matrix that determines the selected measurement points in the iteration; the matrix $\boldsymbol{J}$ is calculated numerically by using finite difference method upon the results from ANSYS.

The iteration procedure stops when the convergence condition is satisfied:

$$
\operatorname{Max}\left\{\left|\frac{b_{i}^{k}-b_{i}^{k-1}}{b_{i}^{k}}\right|\right\}<0.01, \quad i=1,2,3 .
$$

However, this parameter identification procedure is ill-posed due to the existence of measurement errors, especially the aforementioned matching errors. To ensure the well-posedness, an optimal measurement point selection algorithm [5] is incorporated to determine $S$.

Figure $1 \mathrm{~F}$ shows the history of the material parameter identification procedure. The converged value for the levator ani, the left obturator and the right obturator are 0.1264 , 0.1516 and $0.1504 \mathrm{MPa}$, respectively. The final optimal measurement set contains eight measurement points (marked as hollow circles in Figure 1E and dash lines in Figure $1 \mathrm{G}$ ) that mainly locate at the periphery of the levator ani. The finally calculated displacements and the measured displacements are in good agreement not only on the points in the optimal measurement set but also on all the other points. The average difference is $0.29 \mathrm{~mm}$, which is acceptable compared with the resolution of MRI. The 'I'-shaped line segments in Figure $1 \mathrm{G}$ means the range of $\pm 1 \mathrm{~mm}$ around the measured displacement data.

In addition, the parameter identification using all 20 measurement points was also tried. But the identification procedure could not converge. This demonstrates the necessity of incorporating the optimal measurement point selection algorithm.

As the reliable elastic parameters have been obtained, one may use this FE model to calculate the stress distribution. Figure $1 \mathrm{H}$ shows the distribution of von Mises stress at a very high abdominal pressure of $56 \mathrm{cmH}_{2} \mathrm{O}(5.494 \mathrm{kPa})$. 
The high stress intensity at local regions indicates the possibility of prolapse. An intuitive conclusion is that the most fragile regions of the levator ani are: the front-ends connected to the os pelvicum, the middle zone of the upper surface, and the surface junction with the tailbone and two ischial spines. These observations could be valuable for relevant medical studies on the mechanism of PF dysfunctions.

This work was supported by the National Natural Science Foundation of China (11272168).
1 d'Aulignac D, Martins JA, Pires EB, Mascarenhas T, Natal Jorge RM. A shell finite element model of the pelvic floor muscles. Comput Method Biomec, 2005, 8: 339-347

2 Epstein LB, Graham CA, Heit MH. Systemic and vaginal biomechanical properties of women with normal vaginal support and pelvic organ prolapse. Am J Obstet Gynecol, 2007, 197: 165.e1-6

3 Ogden RW. Nonlinear Elastic Deformations. New York: Dover Publications, 1998

4 Xiang ZH, Swoboba G, Cen ZZ. On the optimal layout of displacement measurements for parameter identification process in geomechanics. Int J Geomech, 2003, 3: 205-216

5 Xiang ZH, Wang LQ, Zhou MS. Suppressing damage identification errors from selected natural frequencies and mode shape points. Inverse Probl Sci En, 2012, 20: 871-890

Open Access This article is distributed under the terms of the Creative Commons Attribution License which permits any use, distribution, and reproduction in any medium, provided the original author(s) and source are credited. 